\title{
Soledad y salud: ¿cómo se relacionan y difieren según la edad y la escolaridad? ${ }^{1}$
}

\author{
Loneliness and health: How do they relate \\ and differ in terms of age and schooling?
}

\author{
Daniela J. Cruz-Vargas, Rozzana Sánchez-Aragón y Vanesa Castro-Torres ${ }^{2}$
}

Citación: Cruz-Vargas, D.J., Sánchez-Aragón, R. y Castro-Torres, V. (2020). Soledad y salud: ¿cómo se relacionan y difieren según la edad y la escolaridad? Psicología y Salud, 30(2), 231-241. https://doi.org/10.25009/pys.v30i2.2657.

\section{RESUMEN}

Si bien la disponibilidad de relaciones sociales supone un recurso básico para la promoción de la salud y el bienestar, no son necesariamente un factor protector en razón de que una relación con problemas o carencias es en sí misma una fuente principal de estrés. En particular, estas últimas, sean reales o percibidas, constituyen un sentimiento negativo denominado soledad, comúnmente asociado con depresión, ansiedad, insatisfacción e infelicidad, lo que trae consigo efectos negativos importantes para la salud general. Con base en lo anterior, los propósitos de este estudio fueron identificar el impacto de la soledad en la salud y explorar las posibles diferencias según la edad y la escolaridad. Se empleó una muestra de 387 participantes heterosexuales de la Ciudad de México, con edades de entre 18 y 62 años y con escolaridad mínima de secundaria, a quienes se les aplicaron la Escala para Evaluar Soledad en la Pareja y la Encuesta de Salud SF-36. Algunos de los resultados sobresalientes muestran que experimentar sentimientos de vacío trae consigo una menor vitalidad y más problemas emocionales que afectan el interés por convivir con otras personas, así como dificultades para llevar a cabo las actividades cotidianas. En cuanto a las diferencias, los participantes maduros (45-62 años) reportaron experimentar más problemas de salud que los adultos (31-44) y los jóvenes (17-30). Respecto a su escolaridad, los participantes con mayor grado educativo refirieron más sentimientos de vacío, así como más problemas de salud relacionados con la función y el rol físico.

Palabras clave: Soledad; Salud mental; Grupos etarios; Escolaridad; México.

\begin{abstract}
Although available social relations are a basic resource for the promotion of health and wellbeing, their mere presence is not necessarily a protective factor, since relationships with problems or deficiencies are themselves major sources of stress. Deficiencies - whether real or perceived - lead to a negative feeling of loneliness, commonly associated with symptoms of depression and anxiety, as well as feelings of dissatisfaction and unhappiness which, in turn, affect health in general. In this context, the purpose of the present study was to identify the impact of loneliness on health, and to explore possible differences in these variables by age and schooling. A sample of 388 heterosexual participants from Mexico City, aged 17 to 62 years and a minimum of secondary schooling took two questionnaires: one to assess loneliness in the couple, and the SF-36 Health Survey. The main results show that experiencing feelings of emptiness is associated with less vitality, more emotional problems that affect the interest in living with other people and difficulties in carrying out daily activities. Regarding age differences, mature participants (45-62 years) reported experiencing more health problems followed by adults (31-44) and younger participants (17-30). Regarding schooling, those with higher educational level (bachelor's degree) reported more feelings of emptiness as well as more health problems related to physical and role functioning.
\end{abstract}

Keywords: Loneliness; Mental health; Age groups; Scholarship; Mexico.

\footnotetext{
${ }^{1}$ Investigación realizada gracias al Programa UNAM-PAPIIT IN304919 del proyecto: "Factores protectores y de riesgo a la salud en parejas sanas y con enfermedad crónico-degenerativa". Artículo recibido del 5 de junio y aceptado el 14 de noviembre de 2019.

${ }_{2}^{2}$ Facultad de Psicología, Universidad Nacional Autónoma de México, Av. Universidad 3004, Col. Copilco-Universidad, 04515 Ciudad de México, correos electrónicos: danielacruz.fcpsi@gmail.com, rozzara@unam.mx y vannect7@gmail.com.
} 
$\mathrm{L}$ os seres humanos requerimos satisfacer una gran cantidad de necesidades, algunas de las cuales solo pueden saciar a partir de la respuesta positiva de otros, como el contacto físico, la intimidad, el establecimiento de vínculos y la pertenencia a un grupo (López, Apodaca, Etxebarría, Fuentes y Ortiz, 1998). Las relaciones interpersonales son fundamentales desde el comienzo de la vida (Zupiria, 2000; la primera de ellas se establece con la madre o con el cuidador primario y constituye una de las más importantes en la vida porque funciona como un modelo para entablar nuevas relaciones a largo del tiempo, entre las que destacan las familiares, las de amistad y las de pareja, todas ellas enmarcadas por un proceso que define el nivel de acercamiento e intimidad con matices que varían de acuerdo con las percepciones, metas, sentimientos y suposiciones individuales (Díaz-Loving, 1999).

En tanto que las relaciones familiares constituyen la primera red de apoyo social e influyen en el desarrollo emocional y el moldeamiento de la personalidad, conllevan efectos positivos sobre la autoestima, la aceptación y el cariño por parte de los demás (Musitu y Cava, 2003?); además de que establecen los cimientos para el desarrollo social posterior a través de esquemas mentales, estrategias de resolución de problemas, motivación al logro y reglas y normas a seguir (Valladares, 2008), las de amistad, que se basan en aspectos tales como la confianza, la comunicación, el afecto y el conocimiento mutuo (Giró, 2011). Finalmente, si bien las relaciones de pareja comparten características con las anteriores, como el compromiso adquirido, el reconocimiento público (familia) y el establecimiento voluntario (amistad), difieren de aquellas al basarse en la atracción física y las características de personalidad, así como en la tendencia a depositar en ellas las máximas expectativas $\mathrm{y}$ anhelos, lo que en conjunto hace de este tipo de vinculación la más significativa y la más irreemplazable (Méndez y Dobber, 2005; Ruíz, 2001).

Estas diversas formas de vinculación influyen en el estado de salud y el bienestar de los individuos (Cogollo, Gómez, De Arco, Ruiz y Campo, 2009; Sherman, Vries y Lansford, 2000), ya sea positivamante a través de la satisfacción de ciertos aspectos emocionales, lo que favorece la sensación de acompañamiento, cercanía, superación personal y plenitud (Sherman et al., 2000) y amortigua la influencia perjudicial de factores estresantes al proporcionar recursos informativos, emocionales o tangibles que promueven el comportamiento saludable (Holt-Lunstad, Smith y Layton, 2010), o bien negativamente al generar estados emocionales intensamente perjudiciales, como la frustración y la decepción (Moremen, 2008), con efectos sobre la salud equiparables a los de la hipertensión arterial o la obesidad (House, Landis y Umberson, 1988).

Lo anterior, sugiere que hay un umbral para el contacto social por debajo del cual las personas están en riesgo de enfermar (Cohen, 2004). En este sentido, la perspectiva biopsicosocial considera que la disponibilidad de relaciones sociales cercanas supone un recurso básico para la promoción de la salud y el bienestar (Martos, Pozo y Alonso, 2008; Barra, 2004).

La simple presencia de un compañero no es necesariamente un factor protector, pues una relación problemática o con carencias es en sí misma una fuente de estrés (Kiecolt-Glaser y Wilson, 2017) que aumenta la susceptibilidad a desarrollar enfermedades (Cohen et al., 1998); particularmente las carencias reales o percibidas que se experimentan en las relaciones consideradas íntimas o estrechas constituyen un sentimiento negativo denominado soledad, comúnmente asociada a la depresión, ansiedad, insatisfacción e infelicidad (Cutrona, 1982). Aunque generalmente la soledad ha sido considerada como la ausencia de compañía, alude más bien a una experiencia mucho más compleja (Weiss, 1982), difícil de definir, casi imposible de cuantificar e incluso percibida como vergonzosa (Karnick, 2005).

Fernández, Muratori y Zubieta (2013) afirman que ese déficit puede ser de naturaleza cuantitativa (p. ej., no tener amigos o no tener los que se quisieran) o cualitativa (p. ej., sentir que las relaciones que se tienen son superficiales o insatisfactorias). Por su parte Peplau y Perlman (1982) sostienen que esas carencias pueden ser de tipo afectivo y cognoscitivo; mientras que las primeras se relacionan con la necesidad de intimidad, las segundas lo hacen con la percepción de relaciones sociales por debajo del nivel óptimo esperado en términos de tipo, calidad o cantidad (Peplau, 1985). Partiendo de lo anterior, Dykstra y Fokkema (2007) describen dos perspectivas de estudio: la primera (déficit) asume la sensación displa- 
centera de la carencia de nexos, mientras que la segunda (cognoscitiva) se enfoca en el contraste entre lo esperado y lo obtenido, ya sea en términos de calidad o de la presencia de algún miembro en el entorno. Pese a tales diferencias conceptuales, la soledad posee ciertas características esenciales, entre las que destacan su naturaleza multidimensional (aspectos de personalidad, interacción social y habilidades conductuales), subjetiva (dependiente de la evaluación del individuo) y secuencial (antecedentes, manifestaciones y consecuencias que varían en intensidad y duración) (Montero y Sánchez-Sosa, 2001).

Por su parte, la evidencia empírica sugiere diferencias a partir de características sociodemográficas como el nivel socioeconómico, la escolaridad (Bishop y Martin, 2007; Pinquart y Sörensen, 2001; Savikko, Routasalo, Tilvis, Strandberg y Pitkala, 2005; Theeke, 2009) y la edad (Rokach, 2000). Las dos primeras son consideradas factores que pueden reducir la vulnerabilidad, los sentimientos de desolación, la frustración y la incertidumbre (particularmente el nivel educativo favorece el tamaño de la red social y las relaciones de buena calidad [Cardona, Villamil, Enao y Quintero, 2013]). En cuanto a la edad, Rokach y Neto (2005) sugieren que tanto las circunstancias como los eventos a lo largo de la vida afectarán la forma en que las personas experimentan, evalúan y afrontan las demandas. Lo anterior hace evidente que las causas de la soledad sean muy variables.

Diversos autores (Cardona et al., 2013; Stefani, Seidmann, Pano y Acrich, 2003) apuntan que la soledad trae consigo importantes implicaciones para la salud de los individuos en cuanto que favorece estados negativos tales como la angustia emocional, la tristeza y la desesperanza, que incrementan la activación fisiológica (Hackett, Hamer, Endrigu, Brydon y Steptoe, 2012), produciendo aumentos en la presión arterial, variaciones en los marcadores de inflamación (Shiovitz y Parag, 2018) y deterioro cognitivo, todo ello asociado a la depresión (Donovan et al., 2015), lo que favorece el desarrollo de disfunciones orgánicas, trastornos psicofisiológicos y padecimientos crónicos (Hawkley, Thisted, Masi y Cacioppo, 2010), que en conjunto pueden aumentar hasta en 30\% la mortalidad (Holt-Lunstad, Smith, Baker, Harris y Stephenson, 2015).
Lo anterior resulta sumamente relevante debido a que la salud desempeña un papel esencial en la vida de las personas; por tanto se vuelve una condición indispensable por alcanzar a través del bienestar y la realización de proyectos vitales de las personas (Jara, 2001). Cabe mencionar que la salud también figura como un predictor determinante de la calidad de vida (Sánchez-Aragón, García y Martínez, 2017). Este último concepto ha sido ampliamente explorado debido a que modifica el valor asignado a la vida a partir de la percepción subjetiva relacionada con la satisfacción en cuanto a su situación física, estado emocional y vida familiar, amorosa y social de los individuos.

Aunque esta valoración está influida y moderada por otras personas, es indiscutiblemente personal en virtud de que se hace desde la propia jerarquía de valores. Aunque es un extenso conjunto de factores a evaluar, estos pueden agruparse en general en tres categorías: físico-biológicos (p. ej., discapacidad funcional), emocionales (p. ej., sentimientos de tristeza) y sociales (p. ej., relaciones interpersonales) (Vinaccia y Orozco, 2005). En este marco, se observa que diversos factores, como el estado de salud y psicológico, las relaciones sociales y ciertas características sociodemográficas pueden afectar la calidad de vida de las personas (Botero y Pico, 2007).

Específicamente, la soledad y el estado de salud modifican el valor asignado a la vida en función de la percepción de las limitaciones físicas, psicológicas y sociales; las dificultades para el cumplimiento de metas, expectativas, estándares e intereses (Acosta, Quintana, García y Echeverría, 2017) en áreas consideradas primordiales y significativas, como las relaciones familiares, el apoyo social y el estado funcional; por lo anterior, resulta fundamental identificar la relación entre la soledad y la salud y explorar las posibles diferencias en la soledad y la salud según la edad y la escolaridad.

\section{MÉTODO}

\section{Participantes}

Se trabajó con una muestra no probabilística propositiva (Hernández, Fernández y Baptista, 2014), cuyos criterios de inclusión fueron los de ser ma- 
yores de edad (18 años), con una escolaridad mínima de secundaria, con pareja con la cual se cohabitara y que participara en la investigación, y sin enfermedades crónico-degenerativas. Con base en ello, se reunió un total de 398 adultos heterosexuales de la Ciudad de México, cuya escolaridad se distribuyó de la siguiente manera: secundaria $(18.8 \%)$, bachillerato $(32.1 \%)$ y licenciatura (48.6\%). En cuanto a las edades de los participantes, estas oscilaron entre 17 y 62 años $(M=37.36$, D.E. $=11.16)$. De los 387 participantes que reportaron su estado civil, 207 indicaron estar casados y 180 vivir en unión libre, con un tiempo de la relación de entre 5 meses y 41 años $(M=14.01$, D.E. $=9.6$ años). Se halló una moda de 2 en cuanto al número de hijos.

\section{Instrumentos}

Se aplicó un cuestionario compuesto por dos secciones, la primera de ellas comprendía un apartado en el cual se les preguntaba a los participantes datos sociodemográficos como: sexo, edad, escolaridad, estado civil, tiempo en la relación y número de hijos. En la segunda sección, se encontraban:

\section{Escala de Soledad Social y Emocional para Adultos} (DiTomasso y Spinner, 1993).

Este instrumento evalúa la experiencia subjetiva de displacer, estrés y dolor debidos a las deficiencias percibidas de la interacción socioafectiva o intimidad interpersonal en comparación con las deseadas (Peplau y Perlman, 1982). Consta de 31 reactivos en formato de respuesta tipo Likert de cinco puntos que indican el grado de acuerdo (de 1, totalmente en desacuerdo, a 5, totalmente de acuerdo). En su validación en parejas mexicanas (Granillo y Sánchez-Aragón [en revisión]) evidenció cinco factores: Acompañamiento de la Pareja (p. ej., "Tengo una pareja romántica con quien comparto mis más íntimos pensamientos y sentimientos", "Tengo una pareja romántica a cuya felicidad contribuyo"), Acompañamiento de los amigos (p. ej., "Me siento parte de un grupo de amigos", "Mis amigos entienden mis motivos y mi razonamiento"), Acompañamiento de la familia (p. ej., "Realmente pertenezco a mi familia", "Mi familia realmente se preocupa por mí"), Sentimientos de vacío (p. ej., "Lo que realmente es importante para mí no pa- rece importante para las personas que conozco", "No tengo un amigo(a) que me entienda, pero me gustaría tenerlo(a)"), y Añoranza (p. ej., "Me encuentro deseando a alguien con quien compartir mi vida"). Tales factores explicaron $52.05 \%$ de la varianza total, siendo sus coficientes alfa de Cronbach de $.88, .86, .70, .83$ y .68 , respectivamente.

\section{Cuestionario de Salud SF-36 (Medical Outcomes} Trust, 1996).

Evalúa la salud de la persona a través del autorreporte que, en su versión corta, consta de 19 reactivos tipo Likert de cinco opciones de respuesta que indican el grado de acuerdo con las afirmaciones, distribuidas en seis factores que, en su versión validada en México (cf. Sánchez-Aragón et al., 2017; Zúñiga, Carrillo, Fos, Gandek y Medina, 1999), explican $65.36 \%$ de la varianza y cuentan con coeficientes alfa de Cronbach de moderados a moderadamente altos (de .76 a .95). Los factores que comprende esta medida son, a saber: Función física (p. ej., ¿ "Qué tanto su salud actual le limita para hacer esfuerzos moderados, como mover una mesa, pasar la aspiradora, jugar boliche o caminar más de una hora?”, “¿Qué tanto su salud actual le limita para cargar o llevar la bolsa de compra? ); Rol físico (p. ej., "¿Qué tanto tuvo que dejar de hacer algunas tareas en su trabajo o en sus actividades cotidianas a causa de su salud física?”, “¿Hizo menos de lo que hubiera querido hacer a causa de su salud física?"); Rol emocional y función social (p. ej., “¿Mostró menos interés en convivir con otras personas debido a algún problema emocional, como estar deprimido, triste o nervioso?", "¿Puso menos atención a la hora de estar realizando su trabajo o actividades cotidianas debido a algún problema emocional, como estar deprimido, triste o nervioso?”), Vitalidad (p. ej., “¿Qué tanto se sintió lleno de vitalidad?", “QQué tanto tuvo mucha energía?”), Dolor (p. ej. “¿Tuvo algún dolor por largos periodos de tiempo?”, “¿Qué tanto se sintió cansado?”) y Salud general (p. ej., "Creo que me pongo enfermo más fácilmente que otras personas", "Mi salud es excelente").

\section{Procedimiento}

La aplicación de los cuestionarios fue hecha en su totalidad por cuatro psicólogos que acudieron 
a lugares públicos (plazas comerciales), casas, oficinas y demás para solicitar la participación voluntaria, anónima y confidencial de personas adultas. A cada posible participante se le dijo que los cuestionarios eran parte de una investigación realizada por una institución educativa pública de prestigio en el país, cuyo fin era conocer aspectos relacionados con la salud de las personas, y luego se les indicaban los criterios de inclusión para saber si podían participar en el estudio. Si cumplían con estos, y así lo deseaban, se les proporcionaban los instrumentos para ser llenados en ese mismo momento y lugar, resolviendo sus dudas y poniendo a su disposición los resultados de manera personal.

\section{Análisis de datos}

Inicialmente se realizó una prueba de normalidad Kolmogorov-Smirnov para determinar el tipo de análisis estadístico y correlacionar que había que efectuar; después se llevó a cabo un análisis de correlación de Spearman mediante el cual se identificó el grado de asociación entre la experiencia de la soledad y la salud, y posteriormente la muestra fue dividida por grupos de edad tomando como criterio una distribución equivalente a la de los grupos $(33.3 \%)$, para entonces efectuar una prueba $\mathrm{H}$ de Kruskall-Wallis con el objetivo de conocer si había diferencias significativas entre dichos grupos y su escolaridad.

\section{RESULTADOS}

Inicialmente tuvo que aplicarse la prueba de Kolmogorov-Smirnov con el propósito de verificar si los datos recabados tenían una distribución normal, lo que fue descartado $(p<.001)$, por lo que fue necesario realizar un análisis de correlación de Spearman y así poder cumplir con el primer objetivo. Ya efectuado dicho análisis, las correlaciones, aunque bajas, sugieren que, en la medida en que se tiene una pareja con quien compartir pensamientos y sentimientos íntimos (Acompañamiento pareja), así como amigos para hablar sobre las presiones de la vida (Acompañamiento amigos), se experimenta mayor energía, vitalidad y felicidad (Vitalidad); asimismo, se estima la propia salud como excelente (Salud general) y se tienen menos problemas para realizar actividades físicas, como correr o levantar objetos pesados (Función física) y llevar a cabo tareas domésticas debido a la buena salud física (Rol físico). En particular, a más acompañamiento por parte de la pareja, menos problemas emocionales surgen, como estar triste, deprimido o nervioso, lo que afecta el interés por convivir con otras personas (Rol emocional); a su vez, a más acompañamiento por parte de los amigos, menos dolor corporal se experimenta (Dolor). Por otra parte, a más acompañamiento por parte de la familia (sentir que realmente se pertenece a la familia y que esta se preocupa por uno), más sentimientos de vacío (no tener con quien compartir puntos de vista), más añoranza (encontrarse deseando a alguien con quien compartir la vida y poder decirle que se le ama), más problemas en el rol emocional y físico, dolor y menos vitalidad. Específicamente, a más acompañamiento de la familia y más sentimientos de vacío, más problemas en la función física, y a más acompañamiento familiar, menos salud general (Tabla 1).

Tabla 1. Correlación entre soledad y salud.

\begin{tabular}{|c|l|c|c|c|c|c|c|}
\hline & \multicolumn{1}{|c|}{ Salud } & $\begin{array}{c}\text { Función } \\
\text { física }\end{array}$ & Rol físico & $\begin{array}{c}\text { Rol } \\
\text { emocional }\end{array}$ & Vitalidad & Dolor & $\begin{array}{c}\text { Salud } \\
\text { general }\end{array}$ \\
\hline \multirow{5}{*}{ Soledad } & Acompañamiento de pareja & $-.180 * *$ & $-.123 * *$ & $-.110^{*}$ & $.181 * *$ & $.180 * *$ \\
\cline { 2 - 8 } & Acompañamiento de amigos & $-.219 * *$ & $-.189 * *$ & & $.253 * *$ & $-154 * *$ & $.114 *$ \\
\cline { 2 - 8 } & Acompañamiento familiar & $.167 * *$ & $.248 * *$ & $.287 * *$ & $-.381 * *$ & $.271 * *$ & $-.109 * *$ \\
\cline { 2 - 8 } & Sentimiento de vacío & $.134 *$ & $.272 * *$ & $.303 * *$ & $-.402 * *$ & $.227 * *$ & \\
\cline { 2 - 8 } & Añoranza & & $.160 * *$ & $.307^{*} *$ & $-.353 * *$ & $.146 * *$ & \\
\hline
\end{tabular}

** Correlación significativa a 0.1 (bilateral)

* Correlación significativa a 0.5 (bilateral)

Finalmente, para dar cumplimiento al segundo objetivo, que era el de explorar diferencias en las variables bajo estudio en función de la edad y la escolaridad, se llevaron a cabo dos pruebas de 
Kruskal-Wallis por separado. Para el caso de la edad, la muestra fue dividida (ver el análisis de datos más adelante), y luego de realizar la prueba mencionada, se obtuvieron diferencias significa- tivamente estadísticas en los tres rangos de edad, tanto para los factores de soledad como de salud. Todos los coeficientes se muestran en la Tabla 2.

Tabla 2. Diferencias en soledad y salud por grupos de edad.

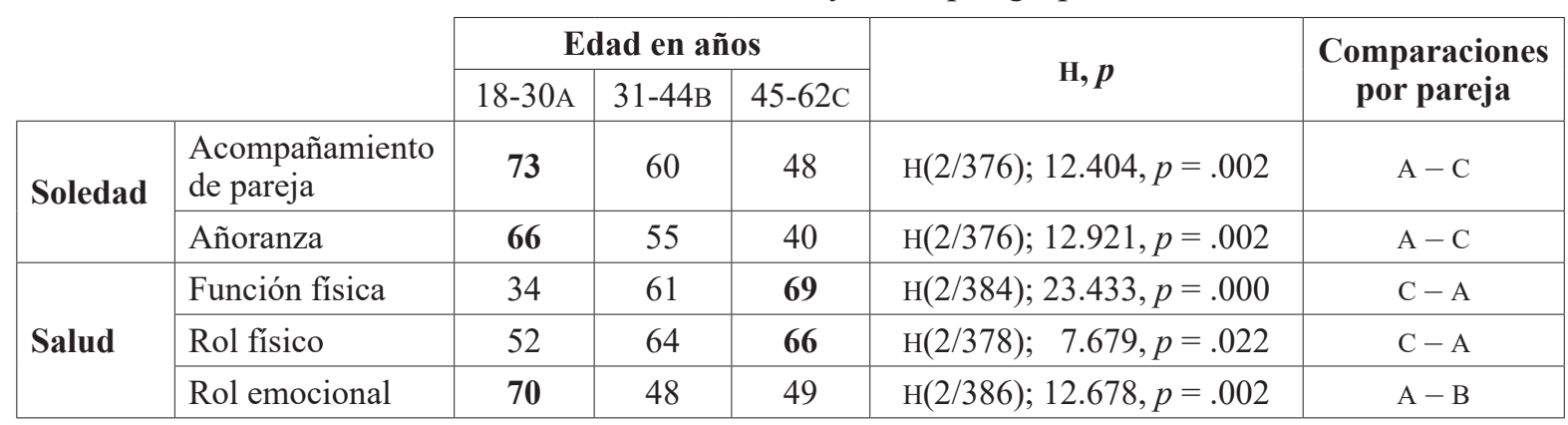

Los resultados muestran que las personas más jóvenes (18-30 años) dijeron sentir más acompañamiento con la pareja, así como mayor añoranza y más problemas con el rol emocional (problemas emocionales como estar triste que pueden afectar el interés por convivir con otras personas), en comparación con las maduras (45-62 años). Aunado a lo anterior, se evidencia que son justamente quienes se hallan en este rango de edad quienes experimentan más problemas con la función (dificultad para realizar tareas cotidianas debido a su salud física) y el rol físico (limitaciones o dificultades para realizar actividades físicas).

En lo que respecta a la escolaridad, se hallaron diferencias estadísticamente significativas, cuyos coeficientes pueden observarse en la Tabla 3.

Tabla 3. Diferencias en soledad y salud por escolaridad.

\begin{tabular}{|l|l|c|c|c|c|c|}
\cline { 2 - 7 } \multicolumn{2}{c|}{} & Sec. & Bach. & Lic. & H, $\boldsymbol{p}$ & $\begin{array}{c}\text { Comparaciones } \\
\text { por pareja }\end{array}$ \\
\hline \multirow{2}{*}{ Soledad } & $\begin{array}{l}\text { Acompañamiento } \\
\text { de amigos }\end{array}$ & $\mathbf{2 0}$ & 56 & 108 & $\mathrm{H}(2 / 379) ; 26.922, p=.000$ & $\mathrm{C}-\mathrm{A}$ \\
\cline { 2 - 7 } & $\begin{array}{l}\text { Acompañamiento } \\
\text { de familia }\end{array}$ & $\mathbf{4 2}$ & 51 & 77 & $\mathrm{H}(2 / 372) ; 7.393, p=.025$ & $\mathrm{C}-\mathrm{A}$ \\
\cline { 2 - 7 } & $\begin{array}{l}\text { Sentimiento } \\
\text { de vacío }\end{array}$ & 47 & 63 & $\mathbf{7 1}$ & $\mathrm{H}(2 / 363) ; 17.358, p=.000$ & $\mathrm{C}-\mathrm{A}$ \\
\hline \multirow{2}{*}{ Salud } & Función física & 47 & 42 & $\mathbf{7 4}$ & $\mathrm{H}(2 / 379) ; 23.550, p=.000$ & $\mathrm{C}-\mathrm{B}$ \\
\cline { 2 - 7 } & Rol físico & 42 & 58 & $\mathbf{8 2}$ & $\mathrm{H}(2 / 373) ; 9.464, p=.009$ & $\mathrm{~A}-\mathrm{B}$ \\
\hline
\end{tabular}

Los hallazgos obtenidos muestran que las personas con menor nivel educativo (secundaria), reportan menor acompañamiento con los amigos y la familia, en comparación con las que tienen licenciatura, siendo así quienes reportan más sentimientos de vacío y más dificultades para ejecutar actividades físicas (Función física) y tareas cotidianas (Rol físico)

\section{DISCUSIÓN}

Fernández, Fernández y Cieza (2010) afirman que la salud de las personas, como seres sociales, de- pende en gran medida de un intercambio interpersonal favorable en términos emocionales y cognoscitivos; al mismo tiempo, el trabajo empírico ha permitido establecer relaciones entre su disponibilidad y la calidad de vida de las personas (Palomar, 2000). Aunque los hallazgos aquí obtenidos muestran correlaciones bajas, resulta posible observar lo anteriormente dicho, ya que a mayor acompañamiento con la pareja y amigos, mayor vitalidad y energía; el estado de salud se estima como excelente y se tienen menos dificultades o limitaciones para realizar tareas domésticas y actividades físicas. En el mismo sentido, un estado óptimo de salud favorece sensaciones de felicidad, 
reduce la ansiedad, la depresión y el estrés, al mismo tiempo que mejora las actitudes hacia sí mismo y el entorno, además de ofrecer oportunidades de socialización que mejoran la interacción (Ministerio de Sanidad, 2015). A este respecto, Furman y Wehner (1994) señalan que se ha considerado que las relaciones - particularmente las románticas y de amistad- contribuyen al desarrollo de la competencia interpersonal, la que genera oportunidades para el enriquecimiento mutuo. Lo anterior, aunado a las repercusiones positivas de las vivencias psicológicas que favorecen conductas adaptativas de salud, como la búsqueda de atención médica, el ejercicio y dieta saludable (Barra, 2004), trae consigo grandes beneficios para la salud y tiene efectos positivos sobre los procesos psicofisiológicos del organismo (Smith, Ruiz y Uchino, 2004), lo que puede verse reflejado en menos dificultades y limitaciones físicas.

Paradójicamente, experimentar el acompañamiento de la familia trae consigo repercusiones relacionadas con la falta de energía, vitalidad y felicidad, problemas emocionales que afectan el interés por relacionarse socialmente y que implican más dificultades en las actividades cotidianas y las físicas, más dolor y una estimación más negativa del estado de salud en general, lo que quizá se deba a que, si bien la familia puede ser fuente de satisfacción, bienestar y soporte afectivo, también impulsa y conduce a la preocupación, el cuidado y la atención de las personas consideradas emocionalmente próximas, lo que entraña el cumplimiento de deberes específicos (Izquieta, 1996); además, exige requerimientos energéticos que podrían generar una disminución de la vitalidad y, por consiguiente, estados físicos desagradables. Al representar un alto valor de significado y compromiso social y personal, las carencias o los conflictos pueden ocasionar profundos desequilibrios emocionales y descompensación en el estado de salud debido a que la familia constituye una fuente principal de apoyo que no puede ser sustituida por ninguna otra (Louro, 2003).

De manera similar a los resultados anteriores, los sentimientos de vacío y añoranza repercuten directamente en la vitalidad y el dolor, así como en las dificultades y limitaciones en las actividades cotidianas y físicas, en razón de que emociones negativas como la tristeza, la soledad, la ansiedad o la depresión coadyuvan al desarrollo de enfermedades psicosomáticas o autoinmunes (Hawkley et al., 2006; Shiovitz y Parag, 2018); asimismo, dichos estados emocionales se asocian comúnmente con trastornos afectivos tales como la depresión, caracterizada por fatiga, falta de iniciativa, apatía y desaliento (Ellis y Grieger, 1981; Instituto Nacional de Neurología, 2017), lo que dificulta la ejecución de las actividades cotidianas, aumenta las sensaciones dolorosas y disminuye los estados positivos, como la felicidad y la vitalidad.

En lo que respecta al segundo objetivo, los resultados muestran que las personas más jóvenes (de 18 a 25 o más años) dijeron contar en mayor medida con una pareja romántica con la que tenían en común los más íntimos y profundos sentimientos, la que las alentaba cuando más lo requerían (acompañamiento con la pareja), o tenían más deseos de encontrar a alguien con quien compartir la vida, y manifestaban una necesidad insatisfecha por una relación romántica cercana (añoranza), aunado a mayores problemas emocionales -como estar triste- que pueden afectar el interés por convivir con otras personas (rol emocional), en comparación con las personas maduras (45-62 años). Tales hallazgos resultan consistentes con lo apuntado por Arnet (2000), quien denomina a dicho periodo "adultez emergente", la cual se caracteriza por el decremento en la cantidad del tiempo dedicado a los amigos (Reis, Lin, Bennett y Nezlek, 1993), la búsqueda y priorización del establecimiento de una relación de pareja que perdure, la adquisición de nuevas responsabilidades adultas y el inicio de nuevos ciclos vitales. En cuanto a los problemas emocionales, Robinson (2012) considera este periodo como el de mayor riesgo para la aparición de las enfermedades mentales relacionadas con la depresión, debido a la experimentación de crisis emocionales, las que incluyen sensaciones de desolación, aislamiento, inadecuación, dudas de sí mismo, miedo al fracaso (Robbins y Wilner, 2008), soledad, confusión y ansiedad. De igual modo, los cambios en el entorno, la sensación de pérdida de relaciones sociales (amigos, familia, maestros), ciclos de vida y responsabilidades pueden generar inestabilidad y estrés (Arnett, 2006).

Por su parte, el acompañamiento más pobre experimentado con la pareja de entre 45 y 62 años 
puede relacionarse con cambios en las funciones sexuales (Minuchin, 1986) y el proceso de separación con los hijos, tras el cual cada miembro de la pareja se enfoca en la búsqueda de satisfacción propia, lo que en conjunto favorece el distanciamiento entre la díada (Sánchez-Aragón, 2016). En lo que respecta a la salud, las dificultades presentes en este periodo se vinculan estrechamente con el decremento en la salud (Organización Mundial de la Salud, 2000), que comienza con la perdida de potencialidades físicas -alrededor de los 50- y luego con el declive del aspecto físico, la salud, el bienestar y la vitalidad (Martínez, Piqueras y Ramos, 2010; Navarro, Meléndez y Tomás, 2008).

En conjunto, estas diferencias etáreas resultan congruentes con lo apuntado por Perlman (1988), quien sugiere que la soledad experimentada en las distintas etapas de la vida se asocia con déficits en diferentes dominios del compromiso social, que durante la edad adulta y media son el parentesco y las relaciones matrimoniales los aspectos más importantes. De manera similar, Victor y Yang (2011) señalan que los adultos de edad media y mayor experimentan la soledad en virtud de los déficits en la calidad de sus compromisos sociales, así como la pérdida de una relación de confianza asociada con el abandono o el deceso del cónyuge.

Finalmente, las diferencias por escolaridad mostraron que las personas con menor nivel educativo (secundaria) reportan tener menos acompañamiento por parte de los amigos y la familia, lo que puede deberse a que la educación formal supone un escenario clave en la interacción social del individuo (Silva, 2007) ya que facilita el aprendizaje de habilidades sociales básicas (Pegalajar y Colmenero, 2017), esenciales para el establecimiento y mantenimiento de relaciones fuera de los lazos familiares (amistosas), y amplía, diversifica, fortalece y enriquece dichos vínculos (Holst, Galicia, Gómez y Degante, 2017); por consiguiente, los bajos niveles escolares suponen escasas habilidades sociales y círculos sociales reducidos o deficientes, lo que favorece la percepción de déficits, ya por la calidad de las relaciones amistosas, ya por el número de amigos que se tienen.

Contrarios a la evidencia empírica existente, los resultados aquí obtenidos muestran que personas con un nivel de licenciatura refieren más sentimientos de vacío y más dificultades para realizar actividades físicas y cotidianas. Para comprender tal hallazgo, resulta necesario considerar lo afirmado por Benavente, Callata y Quispe (2010) respecto a que alcanzar un mayor grado escolar supone eventos potencialmente estresantes, entre los que destacan la competitividad, las exigencias del mercado laboral y académico, el exceso de responsabilidades, la constante evaluación y las presiones familiares, sociales y económicas. Tales eventos conforman un nuevo estilo de vida y modifican los repertorios adaptativos en los que el estrés figura ahora como un elemento constante, lo que genera desgaste y deterioro del cuerpo, aumento de peso, debilitamiento del sistema inmune, ansiedad y falta de interés en las actividades físicas (McEwen y Sapolsky, 2006). En este sentido, Murueta (2016) afirma que los sentimientos de vacío se encuentran estrechamente relacionados con crisis emocionales desencadenadas por el hecho de experimentar frustración de manera continua, y mostrar incapacidad para concretar propósitos individuales y limitaciones para efectuar actividades rutinarias que dejan poco espacio para la creatividad.

Finalmente, es preciso subrayar que, si bien las conclusiones aquí expuestas deben tomarse con mucha cautela, dados los índices estadísticos obtenidos, resultan relevantes ya que muestran congruencia con diversos hallazgos empíricos y teóricos. Asimismo, las discrepancias encontradas pueden sugerir que existen formas indirectas en las que ciertas características sociodemográficas influyen en la salud de las personas. En suma, para trabajos posteriores se sugiere explorar cómo es que algunas de las variables aquí exploradas -y otras más- se comportan en personas con enfermedades crónico-degenerativas y su papel como factores protectores o de riesgo para su desarrollo.

\section{REFERENCIAS}

Acosta Q., C.O., Quintana, J.T., García R. y Echeverría, S. (2017). Soledad, depresión y calidad de vida en adultos mayores mexicanos. Psicología y Salud, 27(2), 179-188. 
Arnett, J.J. (2000). Emerging adulthood: A theory of development from the later teens through the twenties. American Psychologist, 55, 469-480.

Arnett, J.J. (2006). The psychology of emerging adulthood: What is known and what remains to be known. En J. J. Arnett y J. L. Tanner (Eds.): Emerging adults in America. Coming of age in the 21st century (pp. 303-330). Washington, D.C.: American Psychological Association Press.

Barra A., E. (2004). Apoyo social, estrés y salud. Psicología y Salud, 14(2), 237-243.

Benavente, T., Callata, L. y Quispe, P. (2010). Nivel de estrés y estrategias de afrontamiento en estudiantes de la Facultad de Enfermería. Enfermería Global, 4(1), 5-12.

Bishop, A.J. y Martin, P. (2007). The indirect influence of educational attainment on loneliness among unmarried older adults. Journal of Educational Gerontology, 33(10), 897-917.

Botero de M., B.E. y Pico M., M.E. (2007). Calidad de vida relacionada con la salud (CVRS) en adultos mayores de 60 años: una aproximación teórica. Hacia la Promoción de la Salud, 12(1), 11-24.

Cardona J., J., Villamil G., M.M., Henao V., E. y Quintero E, A. (2013). El sentimiento de soledad en adultos. Medicina U.P.B., 32(1), 9-19.

Cogollo, Z., Gómez, E., De Arco, O., Ruiz, I. y Campo A., A. (2009). Asociación entre disfuncionalidad familiar y síntomas depresivos con importancia clínica en estudiantes de Cartagena. Colombia. Revista Colombiana de Psiquiatría, 38(4), 637-644.

Cohen, S. (2004). Social relationships and health. American Psychologist, 59(8), 678-684.

Cohen, S., Frank, E., Doyle, W.J., Skoner, D.P., Rabin, B.S. y Gwaltney Jr., J.M. (1998). Types of stressors that increase susceptibility to the common cold in adults. Health Psychology, 17, 214-223.

Cutrona, C. (1982). Transition to collage: Loneliness and the process of social adjustment. En L. A. Pleplau y D. Perlman (Eds.): Loneliness: A sourcebook of current theory, research and therapy (pp. 291-309). New York: Wiley.

Díaz-Loving, R. (1999). Una teoría biopsicosociocultural de la pareja. En R. Díaz-Loving (Ed.): Antología psicosocial de la pareja (pp. 13-33). México: Miguel Ángel Porrúa.

DiTomasso, E. y Spiner, B. (1993). The development and initial validation of the Social and Emotional Loneliness Scale for Adults (SELSA). Personality and Individual Differences, 14(1), 127-134.

Donovan, N.J., Hsu, D.C., Dagley, A.S., Schultz, A.P., Amariglio, R.E., Mormino, E.C., Okereke, O.I., Rentz, D.M., Johnson, K.A., Sperling, R.A. y Marshall, G.A. (2015). Depressive symptoms and biomarkers of Alzheimer's disease in cognitively normal older adults. Journal of Alzheimer's Disease, 46(1), 63-73.

Dykstra, A.P. y Fokkema, T. (2007). Social and emotional loneliness among divorced and married men a women: Comparing the deficit and cognitive perspectives. Basic and Applied Social Psychology, 29(1), 1-12.

Ellis, A. y Grieger, R. (1981). Manual de Terapia Racional-Emotiva. Bilbao: Desclée De Brouwer.

Fernández L., J.A., Fernández F., M. y Cieza, A. (2010). Los conceptos de calidad de vida, salud y bienestar analizados desde la perspectiva de la Clasificación Internacional del Funcionamiento (CIF). Revista Española de Salud Pública, 84(2), $169-184$.

Fernández, O., Muratori, M. y Zubieta, E. (2013). Bienestar eudaemónico y soledad emocional y social. Boletín de Psicología, $108(1), 7-23$.

Furman, W. y Wehner, E.A. (1994). Romantic views: Toward a theory of adolescent romantic relationships. En R. Montemayor, G. R. Adams y T. P. Gullotta (Eds.): Advances in adolescent development: An annual book series. Personal relationships during adolescence (pp. 168-195). Thousand Oaks, CA: Sage Publications.

Giró, J. (2011). Las amistades y el ocio de los adolescentes, hijos de la inmigración. Papers, Revista de Sociología, 96(1), 77-95.

Granillo V., L.F. y Sánchez-Aragón, R. (en revisión). Medición de la soledad en México y su relación con el apego. Ciencia UAT.

Hackett, R.A., Hamer, M., Endrighi, R., Brydon, L. y Steptoe, A. (2012). Loneliness and stress-related inflammatory and neuroendocrine responses in older men and women. Psychoneuroendocrinology, 37(11), 1801-1809.

Hawkley, C.M., Masi, C.M., Berry, J.D. y Cacioppo, J.T. (2006). Loneliness in a unique predictor of age-related differences in systolic blood pressure. Psychology and Aging, 21(1), 152-164.

Hernández S., R., Fernández C., C. y Baptista L., P. (2014). Metodología de la investigación (6 ed.). México: McGraw-Hill Educador.

Holst M., I.C., Galicia, B.Y., Gómez V., G. y Degante G., A. (2017). Las habilidades sociales y sus diferencias en estudiantes universitarios. Vertientes. Revista Especializada en Ciencias de la Salud, 20(2), 12-21.

Holt-Lunstad, J., Smith, T.B., Baker, M., Harris, T. y Stephenson, D. (2015). Loneliness and social isolation as risk factor for mortality: A meta-analityc review. Perspectives on Psychological Science, 10(2), 227-237.

Holt-Lunstad, J., Smith T.B. y Layton, J.B. (2010). Social relationships and mortality risk: A meta-analysis. Review. PLos Medicine, 7(7), 1-20.

House, J.S., Landis, K.R. y Umberson D. (1988). Social relationships and health. American Association for the Advancement of Science, 241(4865), 540-545. 
Instituto Nacional de Neurología (2017). Depresión. Recuperado de http://www.innn.salud.gob.mx/interna/medica/padecimientos/depresion.html.html.

Izquieta E., J.L. (1996). Protección y ayuda mutua en las redes familiares. Revista Española de Investigaciones Sociológicas, 74(1), 189-208.

Jara N., M.I. (2001). Ética de la salud como ética de la vida. Gerencia y Políticas de Salud, 1(1), 86-102.

Karnick, P.M. (2005). Feeling lonely: Theorical perspectives. Nursing Science Quartely, 18(1), 7-12.

Kiecolt-Glaser, J.K. y Wilson, S.J. (2017). Lovesick: How couple's relationships influence health. The Annual Review of Clinical Psychology, 13(1), 421-443.

López, F., Apodaca, P., Etxebarria, I., Fuentes, M.J. y Ortiz, M.J. (1998). Conducta prosocial en preescolares. Infancia y Aprendizaje, 82(1), 45-61.

Louro B., I. (2003). La familia en la determinación de la salud. Revista Cubana de Salud Pública, 29(1), 48-51.

Martínez G., A.E., Piqueras, J.A. y Ramos L., V. (2010). Inteligencia emocional en la salud física y mental. Electronic Journal of Research in Educational Psychology, 8(2), 861-890.

Martos, M.J., Pozo, C. y Alonso, E. (2008). Influencia de las relaciones interpersonales sobre la salud y la conducta de adherencia en una muestra de pacientes crónicos. Boletín de Psicología, 93(1), 59-77.

McEwen, B. y Sapolsky, R. (2006). El estrés y su salud. Las Hormonas y Usted. Recuperado de: https://academic.oup.com/jcem/ article/91/2/E1/2843212.

Medical Outcomes Trust (1996). Cuestionario General de Salud SF-36. Boston: Autor.

Mejía, R. (2016, mayo 14). "Vacío existencial, un mal contemporáneo" (entrevista con el Dr. Marco Eduardo Murueta). Psicología y Psicoterapia. Recuperado de http://murueta.mx/index.php/textos/64-vacio-existencial-un-mal-contemporaneo.

Méndez C., I. y Dober R., M. (2005). El desarrollo de las relaciones interpersonales en las experiencias transculturales: una aportación del enfoque centrado en la persona. Tesis inédita de maestría. México: Universidad Iberoamericana.

Ministerio de Sanidad (2015). Actividad fisica para la salud y reducción del sedentarismo. Recomendaciones para la población. Madrid: Autor.

Minuchin, S. (1986). Familias y terapia familiar. México: Gedisa.

Montero L., L.M. y Sánchez-Sosa, J.J. (2001). La soledad como fenómeno psicológico: un análisis conceptual. Salud Mental, 24(1), 19-27.

Moremen, R.D. (2008). The downside of friendship: sources of strain in older women's friendships. Journal of Women \& Aging, 20(1), 169-187.

Musitu, G. y Cava, M. (2003). El rol del apoyo social en el ajuste de los adolescentes. Intervención Psicosocial, 12(2), 179-192.

Navarro P., E., Mélendez M., J.C. y Tomás M., J.M. (2008). Relaciones entre variables físicas y de bienestar en la calidad de vida de las personas mayores. Gerokomos, 19(2), 17-21.

Organización Mundial de la Salud (2000). Un enfoque de la salud que abarca la totalidad del ciclo vital. Repercusiones para la capacitación. Ginebra: OMS.

Palomar L., J. (2000). The development of an instrument to measure quality of life in Mexico City. Social Indicators Research, $50(2), 187-208$.

Pegalajar P., M.C. y Colmenero R., M.J. (2017). Actitudes y formación docente hacia la inclusión en educación secundaria obligatoria. Revista Electrónica de Investigación Educativa, 19(1), 84-97.

Peplau, L.A. (1985). Loneliness. En A. Kuper y J. Kuper (Eds.): The Social Science Encyclopedia (p. 474). Boston: Routledge and Kegan Paul.

Peplau, L.A. y Perlman, D. (1982). Perspectives on loneliness. En L. A. Peplau y D. Perlman (Eds.): Loneliness: A sourcerbook of current theory, research and therapy (pp. 1-18). New York: Wiley.

Perlman, D. (1988). Loneliness: a life span, developmental perspective. En R. M. Milardo (Ed.): Families and social networks (pp. 190-220). Newbury Park, CA: Sage.

Pinquart, M. y Sörensen, S. (2010). Influences on loneliness in older adults: a meta-analysis. Journal of Basic and Applied Social Psychology, 23(4), 245-266.

Reis, H., Lin, Y.C., Bennett, M. y Nezlek, J. (1993). Change and consistency in social participation during early adulthood. Developmental Psychology, 29, 633-645. doi: 10.1037/0012-1649.29.4.633.

Robbins, A. y Wilner, A. (2008). Quarterlife crisis: The unique challenges of life in your twenties. New York: Tacher Perigee.

Robinson, O.C. (2012). Development through adulthood: An integrative sourcebook. Basingtoke (UK): Palgrave Macmillan.

Rokach, A. (2000). Loneliness and life cycle. Psychological Reports, 86(1), 629-642.

Rokach, A. y Neto, F. (2005). Age, culture and the antecedents of loneliness. Social Behavior and Personality, 33(5), 477-494.

Ruiz B., D. (2001). Relaciones de pareja. Revista de Educación, 325, 49-57. 
Sánchez-Aragón, R. (2016). La pareja romántica en México. Desentrañando su naturaleza comunicativa y emocional. México: Universidad Nacional Autónoma de México.

Sánchez-Aragón, R., García M., M. y Martínez T., B.D. (2017). Encuesta de Salud SF-36: Validación en tres contextos culturales de México. Revista Iberoamericana de Diagnóstico y Evaluación, 45(3), 5-16.

Savikko, N., Routasalo, P., Tilvis, R.S., Strandberg, T.E. y Pitkälä, K.H. (2005). Predictors and subjective causes of loneliness in an aged population. Archives of Gerontology and Geriatrics, 41(3), 223-233.

Sherman, A.M., Vries, B. y Lansford, J.E. (2000). Friendship in childhood and adulthood: Lesson across the life span. The International Journal of Aging and Human Development, 51(1), 31-51.

Shiovitz, E.S. y Parag, O. (2018). Does loneliness "get under the skin"? Associations of loneliness with subsequent change in inflammatory and metabolic markers. Aging \& Mental Health, 23(10), 1-10.

Silva D., I. (2007). La adolescencia y su interrelación con el entorno. Madrid: Ministerio de Sanidad, Consumo y Bienestar Social. Recuperado de http://www.injuve.es/sites/default/files/LA\%20ADOLESCENCIA\%20y\%20\%20entorno_completo.pdf.

Smith, T.W., Ruiz, J.M. y Uchino, B.N. (2004). Mental activation of supportive ties, hostility, and cardiovascular reactivity stress in Young men and woman. Health Psychology, 23(1), 476-786.

Stefani, D., Sedmann, S., Pano, C. y Acrichi, L. (2003). Los cuidadores familiares de enfermos crónicos: sentimientos de soledad, aislamiento social y estilos de afrontamiento. Revista Latinoamericana de Psicología, 35(1), 55-65.

Theeke, L.A. (2009). Predictors of loneliness in U.S. adults over age sixty-five. Psychiatric Nursing, 23(1), $387-396$.

Valladares G., A.M. (2008). La familia. Una mirada desde la psicología. Revista Electrónica de las Ciencias en Cienfuegos, Medisur, 6(1), 4-13.

Victor, C.R. y Yang, K. (2011). The prevalence of loneliness among adults: a case study of the United Kingdom. The Journal of Psychology Interdisciplinary and Applied, 146(1), 85-104.

Vinaccia, S., Orizco, L.M. (2005). Aspectos psicosociales asociados con la calidad de vida de personas con enfermedades crónicas. Perspectivas en Psicología, 1(2), 125-137.

Weiss, R. (1982). Issues in study of loneliness. En L. A. Peplau y D. Perlman. (Eds.): Loneliness. A sourcebook of current theory, research in therapy (pp. 71-80). New York: Wiley \& Sons.

Zúñiga, M.A., Carrillo J., G.T., Fos, P.J., Gandek, B. y Medina M., M.E. (1999). Evaluación del estado de salud con la Encuesta SF-36: Resultados preliminares en México. Salud Pública de México, 41(2), 110-118.

Zupiria G., X. (2000). Relaciones interpersonales. Generalidades. En G. X. Zupiria (Ed.): Relación entre el profesional de la salud y el enfermo (pp. 9-76). Portugalete: Universidad del País Vasco. 